\title{
Preparation of Cu@Ag Nanoparticles for Conductive Ink
}

\author{
Sijie Wang*, Helong Yu \\ National Engineering Research Center for Mechanical Product Remanufacturing, Army Academy of Armored Forces, Beijing, \\ China \\ Email: *wsj665588@126.com
}

How to cite this paper: Wang, S.J. and $\mathrm{Yu}$, H.L. (2021) Preparation of Cu@Ag Nanoparticles for Conductive Ink. Journal of Materials Science and Chemical Engineering, 9, 1-10.

https://doi.org/10.4236/msce.2021.99001

Received: June 28, 2021

Accepted: September 11, 2021

Published: September 14, 2021

Copyright (c) 2021 by author(s) and Scientific Research Publishing Inc. This work is licensed under the Creative Commons Attribution International License (CC BY 4.0).

http://creativecommons.org/licenses/by/4.0/

(c) (i) Open Access

\begin{abstract}
In order to overcome the shortcomings of low-cost anti-oxidation conductive ink and its preparation method in the field of printing electronics, core-shell coated Cu@Ag nanoparticles were used to prepare conductive ink, and a printed circuit was obtained by inkjet printing. Copper nanoparticles were prepared by a chemical reduction method and then coated with $\mathrm{Cu@Ag} \mathrm{par-}$ ticles by a copper-based self-catalytic reaction. Conductive ink was prepared by ball milling and dispersion and printed on PI film to form a conductive coating. After characterization and analysis, the particle size and dispersion of the obtained Cu@Ag meet the requirements and can be stored stably under normal atmospheric conditions. The resistivity of the conductive film sintered at $300^{\circ} \mathrm{C}$ is only $10.6 \mu \Omega \cdot \mathrm{cm}$.
\end{abstract}

\section{Keywords}

Printed Electronics, Conductive Ink, Core-Shell Nanoparticles, Antioxidant

\section{Introduction}

Printed electronics is a new manufacturing technology for electronic devices based on the principle of printing. It uses functional conductive ink printed directly on a substrate to form conductive circuits or electronic devices. This is a flexible, efficient, environmentally friendly, and inexpensive way of manufacturing materials, which has significant advantages [1]. It not only has had a significant impact on the manufacturing of printed circuit boards (PCB), but also has revolutionized production methods for solar cells, radio frequency identification tags (RFID), flexible circuit boards (FPCB), thin-film transistors, organic light emitting diodes (OLED), and many other applications, and has become a technology hotspot of general interest. 
Printing electronics research in Japan, Korea, the United States, and other countries started early, and ink-jet printing of conductive film prepared by conductive ink has been industrialized. Within this field, the technology of conductive ink with fine precious metal particles such as gold or silver is the most mature and widely used. This kind of conductive ink has good properties but its cost is high, so it is not suitable for mass production, and there is a problem with electron migration using nano-silver ink [2]. Copper is the preferred substitute for gold and silver because of its high conductivity, low price, and low electron mobility [3]. Park et al. [4] synthesized copper particles with a particle size of 40 - $50 \mathrm{~nm}$ by a polyol method and used these particles to prepare conductive ink with good dispersion and low viscosity. The printed coatings exhibit a metallic appearance with resistivity as low as $17.2 \mu \Omega \cdot \mathrm{cm}$. Ida et al. [5] synthesized nano-copper particles coated with gelatin with an average particle size of $46 \mathrm{~nm}$. Conductive films prepared with the conductive ink were treated at either $200^{\circ} \mathrm{C}$ in a $10 \mathrm{ppm} \mathrm{O}_{2}-\mathrm{N}_{2}$ mixed gas flow or at $250^{\circ} \mathrm{C}$ in a 3 vol\% $\mathrm{H}_{2}-\mathrm{N}_{2}$ mixed gas flow, and the resistivity of the films was only $5 \mu \Omega \cdot \mathrm{cm}$. Kubota et al. [6] prepared spherical copper particle conductive ink with a particle diameter of about $18 \mathrm{~nm}$ by continuous supercritical hydrothermal synthesis, and the resistivity of the conductive layer was $16 \mu \Omega \cdot \mathrm{cm}$. Copper inks reported in the literature have good properties, but complex processes and sophisticated instruments are needed to prevent nano-copper from being oxidized in the process of preparation and post-treatment. The oxide resistivity is much higher than that of copper, and it cannot form a sintering neck with surrounding particles. Jeong found that the thicker the oxide layer, the greater the resistance of the copper film [7] by changing the amount of polyvinylpyrrolidone (PVP) added to nano-copper particles with different degrees of oxidation. So far, nano-copper conductive ink cannot be used in industrial production [8] [9].

In this paper, nano-copper particles were prepared by a chemical reduction reaction, and silver-coated nano-copper particles were prepared by a copperbased autocatalytic reaction. Nano Cu@Ag conductive ink which can be stored stably in the air was successfully obtained. Through inkjet printing and sintering, the resistivity of a conductive film can be as low as $10.6 \mu \Omega \cdot \mathrm{cm}$, which can solve the bottleneck problem in industrial production. The work contributes to overcoming the shortcomings of low-cost anti-oxidation conductive ink and its preparation method in the field of printing electronics.

\section{Materials and Methods}

\subsection{Raw Materials and Equipment}

Test materials included anhydrous copper sulfate $\left(\mathrm{CuSO}_{4}, 99.0 \%\right.$, Tianjin Fengchuan Chemical Reagent Technology Co., Ltd.), acacia gum (Select delicate level by hand, Shanghai Aladdin Biochemical Technology Co., Ltd.), Hydrazine hydrate solution $\left(\mathrm{N}_{2} \mathrm{H}_{4} \cdot \mathrm{H}_{2} \mathrm{O}, 80 \%\right.$, Xilong Science Co., Ltd.), acetone $\left(\mathrm{C}_{3} \mathrm{H}_{6} \mathrm{O}\right.$, 99.5\%, Beijing Chemical Plant), absolute ethanol $\left(\mathrm{C}_{2} \mathrm{H}_{6} \mathrm{O}, 99.5 \%\right.$, Beijing Tong- 
guang Fine Chemical Company), acetaldehyde solution $\left(\mathrm{C}_{2} \mathrm{H}_{4} \mathrm{O}, 40 \%\right.$, Tianjin Damao Chemical Reagent Factory), silver nitrate $\left(\mathrm{AgNO}_{3}, 99.0 \%\right.$, Beijing Hengye Cosco Chemical Co., Ltd.), glycerol $\left(\mathrm{C}_{3} \mathrm{H}_{8} \mathrm{O}_{3}, 99.0 \%\right.$, Beijing Hengye Cosco Chemical Co., Ltd.), Spredox D-364 (Taiwan Texas Chemical Company), and Surfynol 465 (Air Products Inc.), BYK-028 (Germany Bike Company).

\subsection{Preparation of Nano-Copper}

Copper nanoparticles were prepared by a chemical reduction method. Copper sulfate was reduced in deionized water with hydrazine hydrate as the reductant and gum arabic as a dispersant. Gum arabic $(0.6 \mathrm{~g})$ and $\mathrm{CuSO}_{4} \cdot 5 \mathrm{H}_{2} \mathrm{O}(0.3 \mathrm{~g})$ were weighed out, and $25 \mathrm{~mL}$ of deionized water were added with stirring until fully dissolved. The mixture was stirred with a magnetic stirrer for $10 \mathrm{~min}$ at $70^{\circ} \mathrm{C}$, then $2.6 \mathrm{~g}$ hydrazine hydrate solution $(80 \%)$ was added and a high-speed disperser was used to stir the mixture for one h. After the reaction, a purple-red colloid was obtained. The prepared nano-copper sol was centrifugally separated $(12,000 \mathrm{rpm})$. The precipitate was washed three times with ethanol and acetone in sequence. A red-brown powder was obtained by drying in a vacuum drying chamber at $40^{\circ} \mathrm{C}$ for $12 \mathrm{~h}$.

\subsection{Preparation of Cu@Ag Nanoparticles}

The purple-red nano-copper sol obtained from the first step was reacted for 10 min with $20 \mathrm{~mL}$ acetaldehyde solution and an excess of hydrazine hydrate in the reaction system. Then $0.2 \mathrm{~g}$ of $\mathrm{AgNO}_{3}$ was dissolved in $20 \mathrm{~mL}$ deionized water, and silver nitrate solution was slowly added. A dark red dispersion was obtained after stirring at room temperature for $20 \mathrm{~h}$ at high speed. The nano Cu@Ag particle dispersion was placed in a high-speed centrifuge, and the precipitate obtained by centrifugal separation $(12,000 \mathrm{rpm})$ was washed three times with ethanol and acetone in sequence. Finally, $\mathrm{Cu@Ag} \mathrm{powder} \mathrm{was} \mathrm{obtained} \mathrm{by} \mathrm{dry-}$ ing in a vacuum drying chamber at $40^{\circ} \mathrm{C}$ for $12 \mathrm{~h}$.

\subsection{Printing and Post-Processing}

With deionized water as solvent, conductive ink with a solids content of $10 \mathrm{wt} \%$ was obtained by high energy ball milling with $0.5 \mathrm{wt} \%$ predox D-364, $3 \mathrm{wt} \%$ glycerol, $0.3 \mathrm{wt} \%$ Surfynol 465 and $0.1 \mathrm{wt} \%$ BYK-028 as additives. Ink-jet printing was done on polyimide (PI) film by an Epson L485 printer and the product was sintered in a vacuum tube furnace for $30 \mathrm{~min}$.

\subsection{Instruments and Characterization}

The phase of the synthesized particles was detected by AXS D8 Advance X-ray diffractometer (Brooke Instruments, Germany). Ultraviolet-visible spectrum analysis was obtained by a Cary 5000 Ultraviolet-visible Spectrophotometer (Agilent Technology Co., Ltd.). The morphology and particle size of the particles were characterized by JEM-2100F transmission electron microscopy (TEM) (JEM- 
2100F) or by a Nanozetasizer-ZS laser particle size analyzer (Malvin Company, UK). The conductivity of the printed layer was tested by a TECH RTS9 fourprobe resistor (Guangzhou Four-probe Technology Co., Ltd.). A Nova NanoSEM 450 Scanning Electron Microscope (FEI) was used to observe the surface morphology of conductive films.

\section{Results and Discussion}

\subsection{Preparation and Characterization of Copper Nanoparticles}

In this experiment, hydrazine hydrate was used as a reductant to quickly reduce copper ions in solution to elemental copper, and no new impurities were introduced into the reaction system, as shown in Equation (1). As the reaction proceeds, the initially formed copper reacts reversibly with unreacted $\mathrm{Cu}^{2+}$ (2). In order to inhibit the occurrence of reaction (2), an excess of hydrazine hydrate was used in this experiment. This also protects nano-copper from oxidation in solution.

$$
\begin{gathered}
\mathrm{N}_{2} \mathrm{H}_{5}^{+}+2 \mathrm{Cu}^{2+} \rightarrow 2 \mathrm{Cu}+\mathrm{N}_{2}+5 \mathrm{H}^{+} \\
\mathrm{Cu}^{2+}+\mathrm{Cu} \rightarrow 2 \mathrm{Cu}^{+}
\end{gathered}
$$

The XRD and UV-vis diagrams of synthesized nano-copper are shown in Figure 1. In Figure 1(a), only three distinct diffraction peaks appear at $43.34^{\circ}$, $50.48^{\circ}$, and $74.18^{\circ}$, which correspond to the (111), (200), and (220) crystal planes of face-centered cubic copper. Figure $1(b)$ is the ultraviolet-visible absorption spectrum of nano-copper sol. There is an obvious plasma resonance absorption peak at $580 \mathrm{~nm}$ in the visible region, which is the characteristic peak of copper. No other impurity peaks appeared, which proves that nano-copper was prepared. Figure 2 shows a TEM image and the DLS particle size distribution of the prepared nano-copper powder. TEM images show that the prepared nano-copper samples have small particle size and spherical shape, and their size distribution ranges from 11 to $34 \mathrm{~nm}$, which indicates that gum arabic has a good protective effect on nano-copper.

\subsection{Preparation and Characterization of Silver Coating}

When $\mathrm{AgNO}_{3}$ solution is added to the prepared nano-copper sol, the substitution reaction $\mathrm{Cu}+2 \mathrm{Ag}^{+}=\mathrm{Cu}^{2+}+2 \mathrm{Ag}$ takes place to obtain a silver coating on the surface of the nano-copper. Because of the difference in standard redox potential between silver $\left(\mathrm{Ag}^{+}+\mathrm{e}^{-} \rightarrow \mathrm{Ag}, \mathrm{E}^{0}=0.779 \mathrm{~V}\right)$ and copper $\left(\mathrm{Cu}^{2+}+2 \mathrm{e}^{-} \rightarrow\right.$ $\mathrm{Cu}, \mathrm{E}^{0}=0.46 \mathrm{~V}$ ), electrons are transferred from $\mathrm{Cu}^{0}$ to $\mathrm{Ag}$ ions, and silver is replaced. In the reaction, one copper atom can produce two silver atoms, so theoretically a dense silver-coated layer can be obtained. The morphology of the product is similar to that of the previously prepared copper core, and the particle size increases slightly. When an excess of hydrazine hydrate is used to prepare nano-copper sol, the standard reduction potential difference between hydrazine hydrate $\left(\mathrm{N}_{2} \mathrm{H}_{4}+4 \mathrm{OH}^{-} \rightarrow \mathrm{N}_{2}+4 \mathrm{H}_{2} \mathrm{O}+4 \mathrm{e}^{-}, \mathrm{E}^{0}=-1.16 \mathrm{~V}\right)$ and silver is about $1.96 \mathrm{~V}$, 


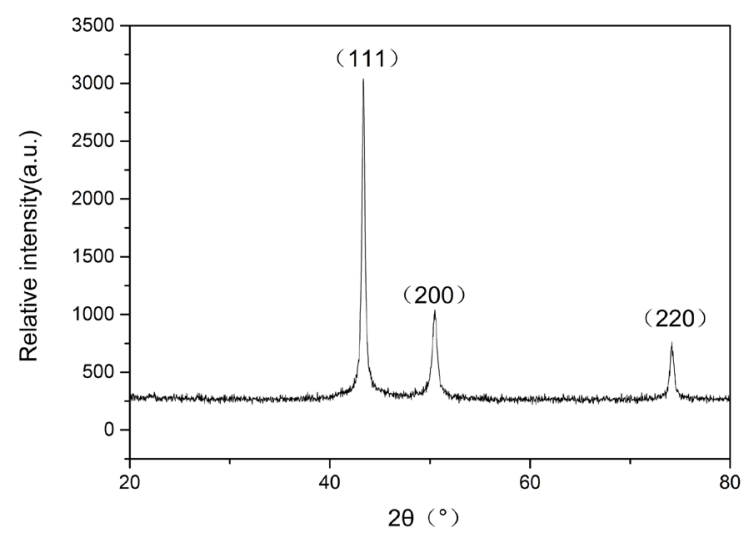

(a)

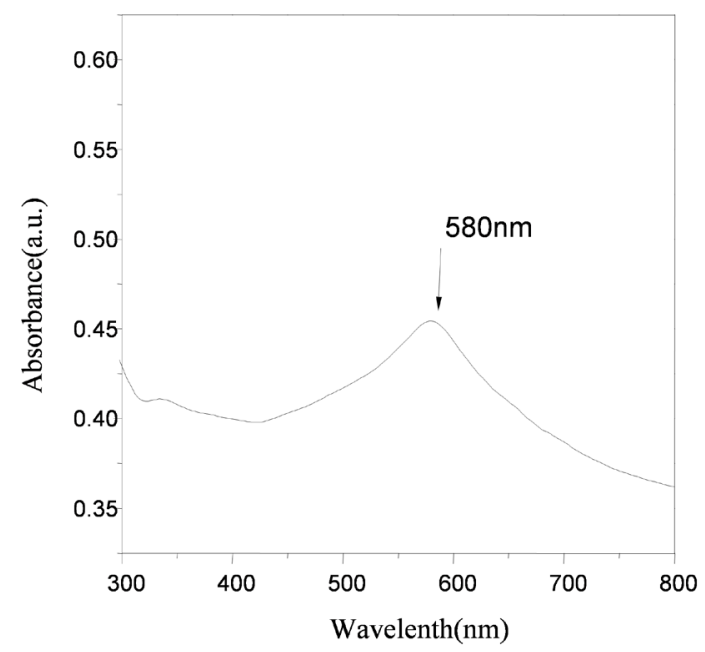

(b)

Figure 1. (a) XRD and (b) UV-vis spectra of nano-copper.
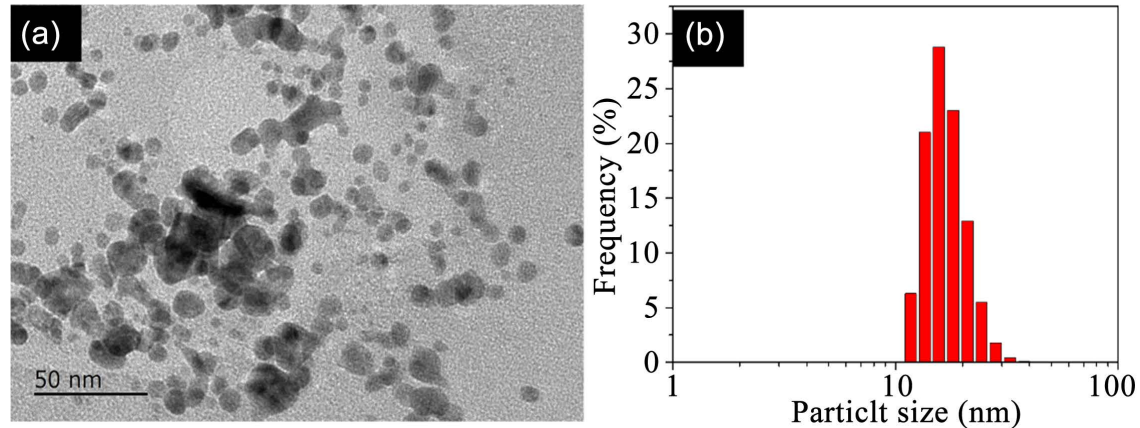

Figure 2. (a) TEM image and (b) particle size distribution of nano-copper.

which is much higher than that between silver and copper. Therefore the products obtained by directly adding silver nitrate solution are silver particles and a small amount of silver-coated copper powder. In order to prevent silver nitrate from being reduced directly by excessive hydrazine hydrate, the excess hydrazine hydrate is consumed by adding acetaldehyde solution $\left(\mathrm{C}_{2} \mathrm{H}_{4} \mathrm{O}+\mathrm{N}_{2} \mathrm{H}_{4} \rightarrow \mathrm{C}_{2} \mathrm{H}_{6} \mathrm{~N}_{2}\right.$ $+\mathrm{H}_{2} \mathrm{O}$ ) to the reaction system before adding the silver nitrate solution. 
Figure 3 is the ultraviolet-visible absorption spectrum of $\mathrm{Cu} @ \mathrm{Ag}$ sol. Not only does the plasma resonance peak of nano-copper appear near $590 \mathrm{~nm}$ in the ultraviolet region, but also the characteristic absorption peak of silver appears near $450 \mathrm{~nm}$. It can be seen that the characteristic peaks of silver in the figure are significantly stronger than those of copper. A possible reason is that silver is coated on the surface of the copper. TEM images of the above-mentioned Cu@Ag particles are shown in Figure 4(a). The morphology and particle size of the Cu@Ag particles are not significantly different from those of the previously prepared nano-copper particles. As shown in Figure 4(b), the silver (111), (200), and (220) rings and the copper (220) and (311) rings are obtained by selective electron diffraction of the sample, but the rings look blurred. The reason for this is that the lattice constants of copper and silver are different, the distribution of the interface is discontinuous, and the optimum diffraction conditions for copper and silver are different. Therefore, some diffraction rings are not clear when we see the copper and silver diffraction rings at the same time, and some crystal planes do not have diffraction rings [10].

In order to explore the effect of different reaction conditions on the silver coating effect, Cu@Ag particles were prepared by controlling the reaction temperature and the mass ratio of copper to silver. The products were placed in air for one week and then analyzed by XRD. The XRD pattern is shown in Figure 5.

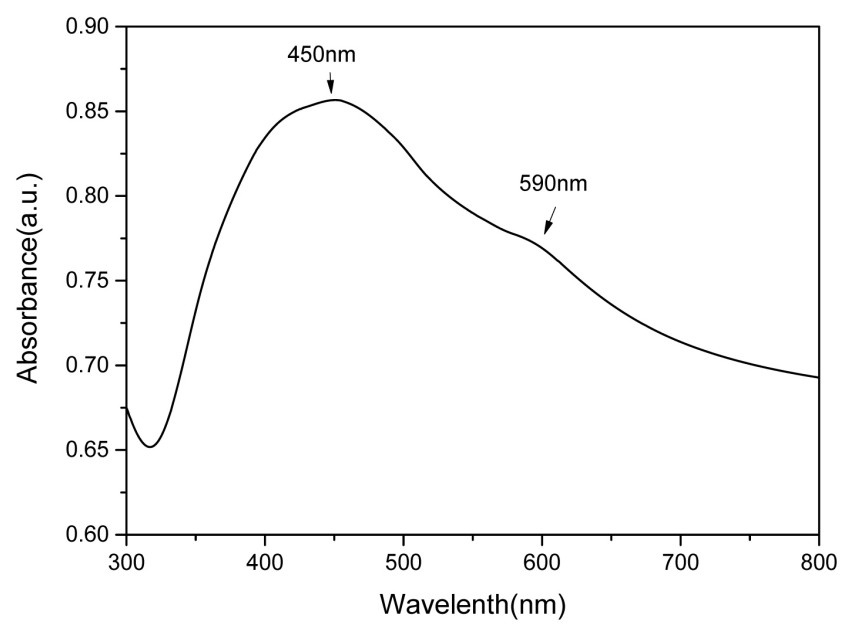

Figure 3. UV-vis spectrum of nano-Cu@Ag particles.
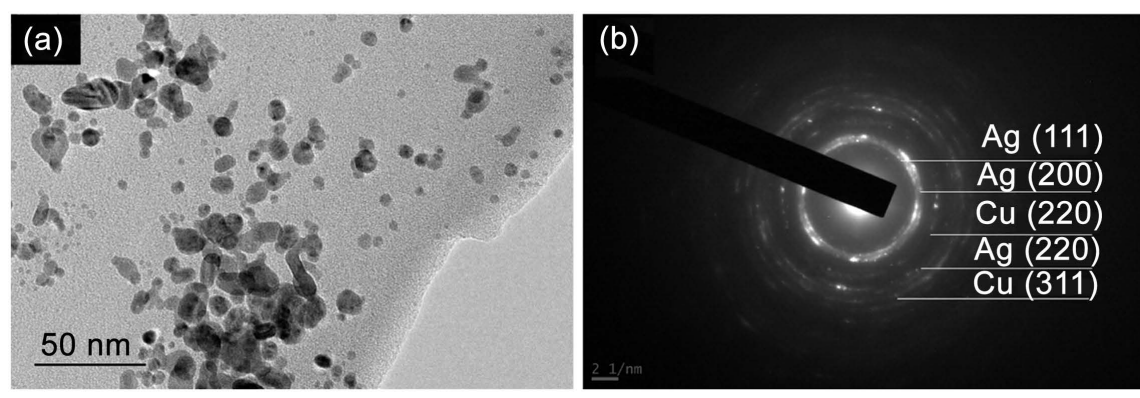

Figure 4. (a) TEM image and (b) selected electron diffraction of nano-Cu@Ag particles. 

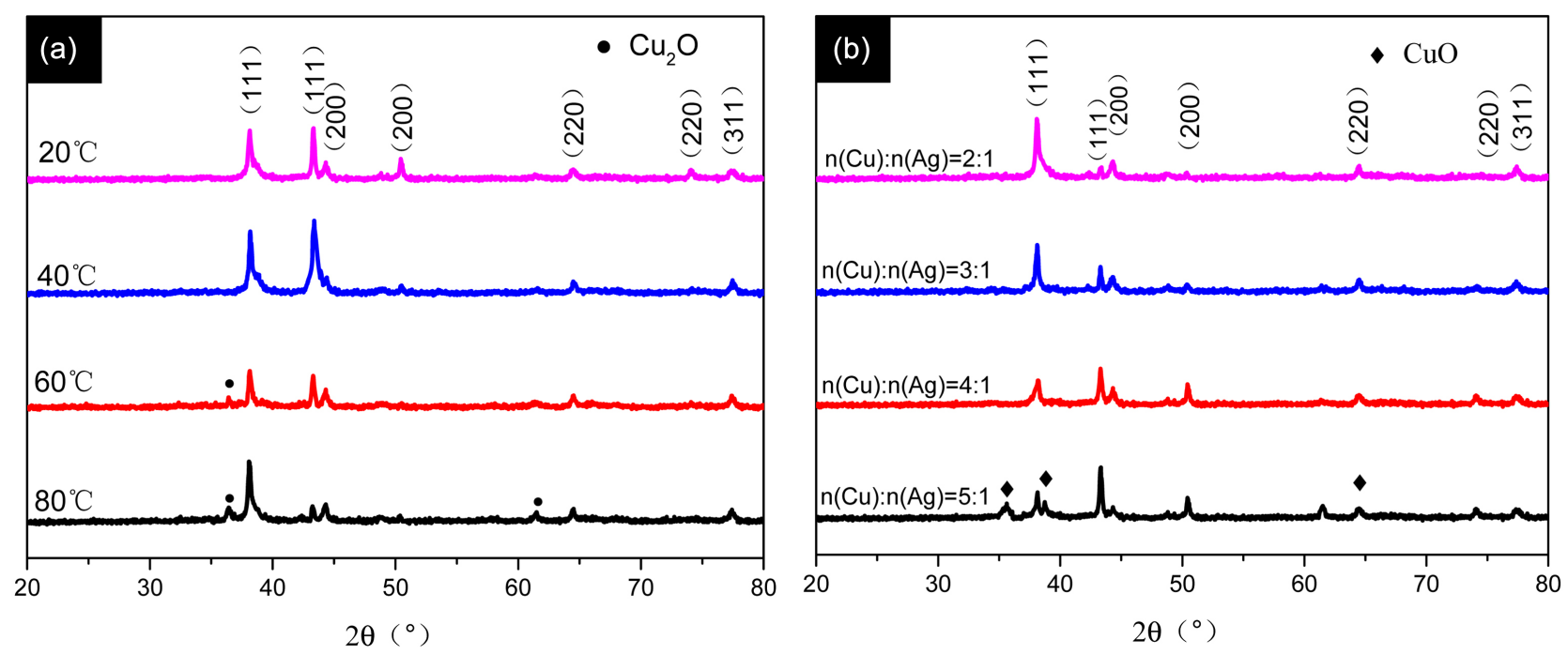

Figure 5. XRD patterns of the phase of nano-Cu@Ag particles with different temperatures and amount of silver.

The results show that the products have diffraction peaks at $43.34^{\circ}, 50.48^{\circ}$, and $74.18^{\circ}$, corresponding to the (111), (200), and (220) faces of face-centered cubic copper, respectively. Diffraction peaks appeared at $38.12^{\circ}, 44.36^{\circ}, 64.43^{\circ}$, and $77.41^{\circ}$, corresponding to the (111), (200), (220), and (311) faces of face-centered cubic silver, respectively. In Figure 5(a), when an excess of silver nitrate is added, the diffraction peak of $\mathrm{Cu}_{2} \mathrm{O}$ appears when the reaction temperature reaches $60^{\circ} \mathrm{C}$, and the diffraction peak of $\mathrm{Cu}_{2} \mathrm{O}$ increases gradually with an increase in the reaction temperature. This is because when the reaction temperature is high, the reaction rate is very fast, and silver ions are rapidly reduced to silver, but they cannot be well deposited on the surface of the copper core, resulting in an incomplete and compact coating. The uncoated copper atoms react with the $\mathrm{Cu}^{2+}$ produced by the displacement reaction: $\mathrm{Cu}^{2+}+\mathrm{Cu}=2 \mathrm{Cu}^{+}$, while free $\mathrm{Cu}^{+}$ reacts with $\mathrm{OH}^{-}$in the solvent to produce $\mathrm{Cu}_{2} \mathrm{O}$, which results in the formation of impurities. Therefore, the reaction is more suitable for our purposes at $20^{\circ} \mathrm{C}$. As indicated in Figure 5(b), when the reaction temperature was $20^{\circ} \mathrm{C}$ and the ratio of copper to silver was 5:1, $\mathrm{CuO}$ was detected one week after the product was produced, which indicates that the silver coating cannot protect the copper core well and the coating effect is not good. However, when the ratio of copper to silver was 4:1, 3:1, or 2:1, no oxide was detected after one week, which indicates that the prepared $\mathrm{Cu@Ag} \mathrm{nanoparticles} \mathrm{had} \mathrm{a} \mathrm{good} \mathrm{coating} \mathrm{structure.} \mathrm{Par-}$ ticles with a good coating effect can be obtained when the atomic ratio of copper to silver is $4: 1$, but the cost will increase if the amount of silver nitrate is increased. Therefore, the appropriate amount of silver nitrate to add is a ratio of copper to silver of 4:1.

\subsection{Characterization of Conductive Films}

Conductive ink was printed on PI film by inkjet printing. After vacuum heating for $30 \mathrm{~min}$, the resistivity of the conductive lines formed at different tempera- 
tures of the coating was measured by a four-probe tester. As shown in Figure 6, with an increase of post-processing temperature, the resistivity of conductive lines decreases continuously, and the conductivity increases. The resistivity is $682.1 \mu \Omega \cdot \mathrm{cm}$ when the post-treatment temperature is $100^{\circ} \mathrm{C}$. When the posttreatment temperature is $150^{\circ} \mathrm{C}$, the conductivity of the sample is greatly improved and the resistivity decreases to $98.6 \mu \Omega \cdot \mathrm{cm}$; at $250^{\circ} \mathrm{C}$ the resistivity is only $14.8 \mu \Omega \cdot \mathrm{cm}$. The resistivity decreases slightly with increasing sintering temperature, reaching $10.6 \mu \Omega \cdot \mathrm{cm}$ at $300^{\circ} \mathrm{C}$. The reason for the increase in conductivity of the conductive circuit may be that the organic additives in ink volatilize during sintering of the nano-Cu@Ag particles, and the adjacent particles form a continuous conductive path in the melted state. With an increase in temperature, the conductive path increases gradually, and the electrons can be conducted more freely in the conductive circuit.

According to the first law of diffusion, the definition of diffusion coefficient $D$, which is the key factor affecting diffusion rate, is as follows: $D=\exp \left(-\frac{Q}{R T}\right) D_{0}$, where $Q$ is the diffusion activation energy, $R$ is the gas constant, $T$ is the thermodynamic temperature, and $D_{0}$ is the diffusion constant. It can be seen that the diffusion coefficient increases with an increase in temperature. That is to say, with an increase of temperature, the diffusion coefficient increases and the degree of fusion between particles increases. In order to verify that nano- $\mathrm{Cu} @ \mathrm{Ag}$ particles melt into continuous thin films after heat treatment, scanning electron microscopy (SEM) was used to characterize the micro-morphology of conductive circuits at different temperatures. As shown in Figure 7, after high temperature treatment at $150^{\circ} \mathrm{C}$, the particles begin to melt and sintering neck appears. Metal atoms diffuse from unstable grain boundary positions to form sintering neck through diffusion.

After sintering at $200^{\circ} \mathrm{C}$, the sintering neck size increases, and the number increases, volume shrinkage is not obvious, the atom diffusion channel widens, and more continuous seepage paths are generated in the circuit, which is consistent with the trend of resistivity reduction shown in Figure 6. When the sintering

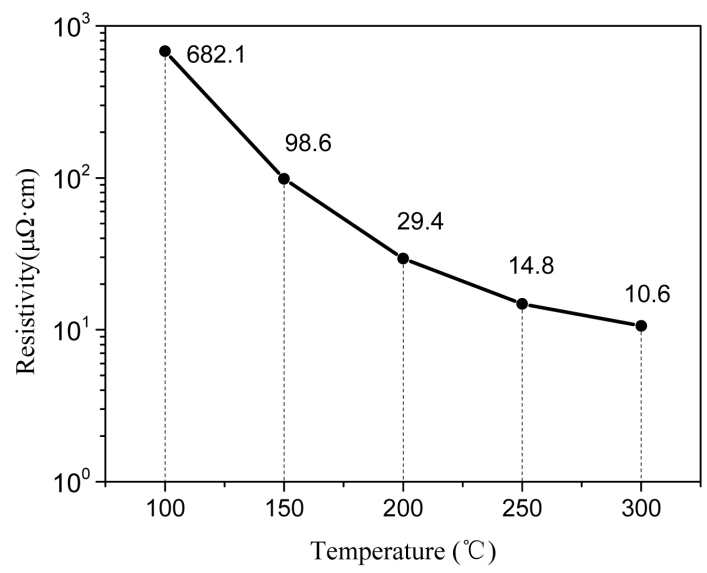

Figure 6. Diagram of resistivity variation with sintering temperature. 

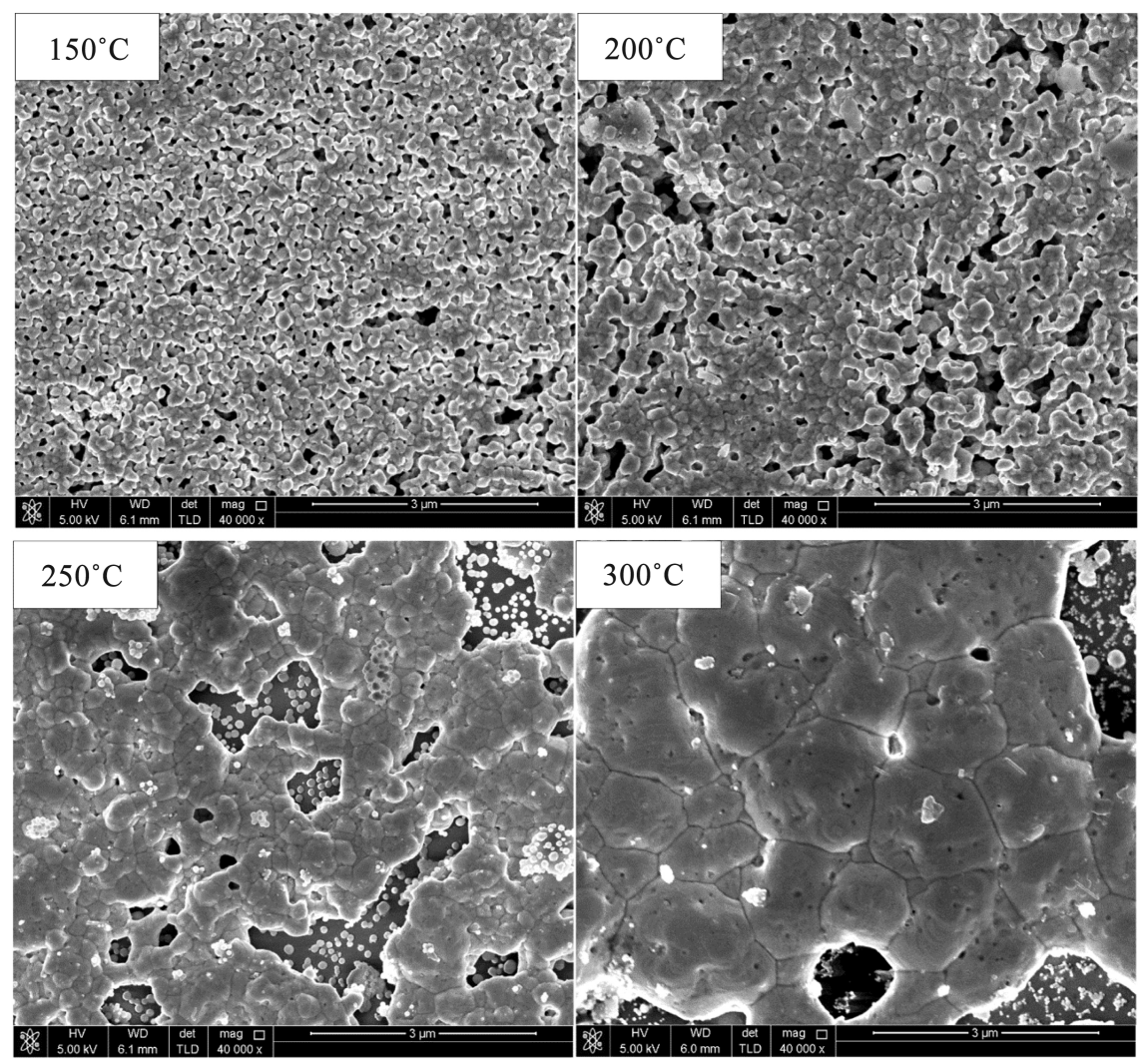

Figure 7. Micromorphology of printed circuits at different post-processing temperatures.

temperature reaches $250^{\circ} \mathrm{C}$, the size and density of the sintering neck increase greatly, the particles connect with each other, the volume shrinks, and the resistivity decreases significantly. When the sintering temperature reaches $300^{\circ} \mathrm{C}$, the particles fuse with each other to form a highly dense conductive layer. It is also shown that the conductivity of the conductive film cannot reach the level of bulk materials because of the large number of voids generated by gaps between the original particles and inhomogeneous volume shrinkage after the temperature reaches $250^{\circ} \mathrm{C}$.

\section{Conclusion}

Copper nanoparticles with high purity and good dispersion were prepared using a liquid phase chemical reduction method. The particle size distribution ranged from 11 to $34 \mathrm{~nm}$. By controlling the amount of silver nitrate and the reaction temperature, Cu@Ag nanoparticles with complete coating and good oxidation resistance were obtained. After sintering, the electrical conductivity improves after sintering, with higher values obtained at higher sintering temperature up to $300^{\circ} \mathrm{C}$, and the resistivity was as low as $10.6 \mu \Omega \cdot \mathrm{cm}$ at $300^{\circ} \mathrm{C}$, which has great potential for several applications.

\section{Acknowledgements}

This research was supported by the National Key R\&D Program of China (Grant 
No. 2018YFB0407401).

\section{Conflicts of Interest}

The authors declare no conflicts of interest regarding the publication of this paper.

\section{References}

[1] Kamyshny, A. and Magdassi, S. (2017) Metallic Nanoinks for Inkjet Printing of Conductive 2D and 3D Structures. In: Magdassi, S. and Kamyshny, A., Eds., Nanomaterials for $2 \mathrm{D}$ and $3 \mathrm{D}$ Printing. Wiley-VCH, Weinheim, Germany. https://doi.org/10.1002/9783527685790.ch7

[2] Yang, W., Liu, C., Zhang, Z., et al. (2013) Preparation and Conductive Mechanism of Copper Nanoparticles Ink. Journal of Materials Science Materials in Electronics, 24, 5175-5182. https://doi.org/10.1007/s10854-013-1541-3

[3] Tam, S.K., Fung, K.Y., Poon, G.S.H., et al. (2016) Product Design: Metal Nanoparticle-Based Conductive Inkjet Inks. Aiche Journal, 62, 2740-2753. https://doi.org/10.1002/aic.15271

[4] Park, B.K., Kim, D., Jeong, S., et al. (2007) Direct Writing of Copper Conductive Patterns by Ink-Jet Printing. Thin Solid Films, 515, 7706-7711.

https://doi.org/10.1016/j.tsf.2006.11.142

[5] Ida, K., Tomonari, M., Sugiyama, Y., et al. (2012) Behavior of Cu Nanoparticles Ink under Reductive Calcination for Fabrication of $\mathrm{Cu}$ Conductive Film. Thin Solid Films, 520, 2789-2793. https://doi.org/10.1016/j.tsf.2011.12.024

[6] Kubota, S., Morioka, T., Takesue, M., et al. (2014) Continuous Supercritical Hydrothermal Synthesis of Dispersible Zero-Valent Copper Nanoparticles for Ink Applications in Printed Electronics. Journal of Supercritical Fluids, 86, 33-40. https://doi.org/10.1016/j.supflu.2013.11.013

[7] Jeong, S., Woo, K., Kim, D., et al. (2010) Controlling the Thickness of the Surface Oxide Layer on $\mathrm{Cu}$ Nanoparticles for the Fabrication of Conductive Structures by Ink-Jet Printing. Advanced Functional Materials, 18, 679-686.

https://doi.org/10.1002/adfm.200700902

[8] Venkata, K.R.R., Venkata, A.K., Karthik, P.S., et al. (2015) Conductive Silver Inks and Their Applications in Printed and Flexible Electronics. RSC Advances, 5, 7776077790. https://doi.org/10.1039/C5RA12013F

[9] Öhlund, T., Schuppert, A.K., Hummelgård, M., et al. (2015) Inkjet Fabrication of Copper Patterns for Flexible Electronics: Using Paper with Active Precoatings. ACS Applied Materials \& Interfaces, 7, 18273-18282.

https://doi.org/10.1021/acsami.5b03061

[10] Anderson, T.S., Iii, R.H.M., Wittig, J.E., et al. (2000) Fabrication of Cu-coated Ag nanocrystals in silica by sequential ion implantation. Nuclear Instruments \& $\mathrm{Me}$ thods in Physics Research Section B: Beam Interactions with Materials and Atoms, 171, 401-405. https://doi.org/10.1016/S0168-583X(00)00369-4 